\title{
Simmel et l'approche sociologique de l'environnement
}

\section{Avertissement}

Le contenu de ce site relève de la législation belge sur la propriété intellectuelle et est la propriété exclusive de l'éditeur.

Les œuvres figurant sur ce site peuvent être consultées et reproduites sur un support papier ou numérique sous réserve qu'elles soient strictement réservées à un usage soit personnel, soit scientifique ou pédagogique excluant toute exploitation commerciale. La reproduction devra obligatoirement mentionner l'éditeur, le nom de la revue, l'auteur et la référence du document.

Toute autre reproduction est interdite sauf accord préalable de l'éditeur, en dehors des cas prévus par la législation en vigueur en Belgique.

\section{Référence électronique}

Philippe Boudes (2008), «Simmel et l'approche sociologique de l'environnement », Emulations. URL : http://shortlinks.revue-emulations.net/boudes

Éditeur : Émulations - Revue des jeunes chercheurs en sciences sociales http://www.revue-emulations.net 


\title{
Simmel et l'approche sociologique de l'environnement
}

Philippe Boudes $^{1}$

\begin{abstract}
Mots-clés : Georg Simmel, sociologie de l'environnement, écologie humaine, morphologie sociale Résumé : Les travaux de Georg Simmel regorgent de réflexions sur les interactions entre phénomènes naturels et sociaux lesquelles caractérisent la question contemporaine de l'environnement. Est-ce pour autant un pionnier de l'approche sociologique de l'environnement ? Si l'on considère les apports conceptuels du sociologue allemand, notamment à travers son approche des formes sociales, de l'espace mais encore de la nature, il est clair que la pensée de Simmel peut étayer certains développements de la sociologie de l'environnement. D'ailleurs, cet article montre également que Simmel a influencé les deux principaux courants fondateurs de la sociologie de l'environnement, l'écologie humaine étasunienne et la morphologie sociale française. En retraçant l'apport cognitif et socio-historique de Simmel pour la sociologie de l'environnement, on contribue à actualiser les études simmeliennes tout en favorisant l'institutionnalisation de la sociologie de l'environnement.
\end{abstract}

Key-words: Georg Simmel, environmental sociology, human ecology, social morphology

Abstract: Simmel's work shows an abundance of reflections on interactions between natural and social phenomena, interactions which are characteristic of environment's contemporary questions. Does it mean that he is a pioneer in environment's sociological study? If we consider that German sociologist's conceptual contributions, notably through his approach of social forms, of space and also of nature, it is clear that Simmel thought can support some developpements in environmental sociology. Meanwbile, this article shows that Simmel influenced both of the founder streams in environmental sociology, U.S. human ecology and French social morphology. Recounting Simmel's cognitive and socio-historical contribution for environmental sociology, we will participate in actualising simmelian studies while favouring the institutionalisation of environmental sociology.

\section{Introduction}

Proposer de revenir sur la pensée de G. Simmel (1858-1918), c'est notamment accepter de confronter ce sociologue allemand aux questions contemporaines et de montrer en quoi ses analyses peuvent être heuristiques encore aujourd'hui. Parmi les questions actuelles, l'environnement est bel et bien une thématique de recherche incontournable pour les sociologues. Après les questions politique et industrielle des siècles précédents, c'est la question environnementale qui domine la fin du XXème et ce début de XXIème siècle (Moscovici, 1968, 2002). Il est pourtant remarquable que les commentateurs de Simmel n'offrent que peu ou pas de place à l'actualité de sa vision de la nature et de l'environnement : ainsi les ouvrages de Léger (1989), Vandenberghe (2000) ou Watier (2003) n'invitent pas à revenir sur cet aspect pourtant caractéristique de l'œuvre simmelienne. Et les travaux des sociologues de l'environnement font écho à cet oubli : alors que les relectures des classiques vont bon train, avec en premier lieu celles de Durkheim (e.g. Catton, 2002 ; Rosa \& Richter, 2008) et Marx (e.g. Foster, 1999, Järvikoski, 1996), de Weber (e.g. West, 1984 ; Murphy, 1994) ou à moindre échelle Le Play (Kalaora \& Savoye, 1996), Halbwachs (Boudes, 2007) ou Veblen (Mitchell, 2001), un seul chercheur à notre

\footnotetext{
${ }^{1}$ Chercheur associé au LADYSS-CNRS, Nanterre, France.
}

Émulations - Revue des jeunes chercheurs en sciences sociales 
connaissance s'est penché sur la question du lien entre l'approche sociologique de l'environnement et la pensée de Simmel (Gross, 2000, 2001).

Cet article s'inscrit dans ce double objectif de contribuer à l'étude de Simmel et à l'analyse sociologique de l'environnement, dans le prolongement de l'entreprise initiée par Gross. En effet, si Simmel aborde de nombreux aspects de la réalité sociale, il n'en néglige pas moins d'intégrer dans sa réflexion l'influence des phénomènes non-sociaux. De même, puisque la (jeune) sociologie de l'environnement veut être reconnue au même titre que les autres domaines sociologiques, elle doit conforter son assise en se confrontant aux textes fondateurs de la sociologie. Il ne s'agit pas pour autant de faire du sociologue allemand un savant à l'intuition exagérément prospective, ni d'affirmer que l'environnement doit nécessairement avoir été abordée par les classiques pour être légitime; c'est d'abord l'approfondissement de l'analyse des interactions entre phénomènes naturels et sociaux qui pousse à lier cet auteur et cette thématique.

Dans tous les cas, il est clair que le regard nouveau que les tenants d'une approche sociologique de l'environnement peuvent porter sur les travaux de Simmel contribue à approfondir les études simmeliennes d'une part, socio-environnementales d'autre part. Si l'on se borne à relire ne serait-ce que les premiers travaux de sociologie de Simmel, on ne peut qu'être frappé par le champ lexical utilisé par le sociologue allemand qui évoque non seulement l’inscription matérielle des phénomènes sociaux mais encore la dépendance de ceux-ci à l'égard des formes qu'ils prennent et à l'égard des facteurs nonsociaux dont les facteurs naturels. Sa vision même de la sociologie, axée sur les formes des associations humaines en tant qu'elles sont la matière du processus social (Simmel, 1894 : 499) accepte dès le départ de s'ouvrir à des déterminations de natures diverses.

\begin{abstract}
«Dans les groupes sociaux, écrit-il, que leurs buts et leurs caractères moraux font aussi différents qu'on peut l'imaginer, nous trouvons par exemple les mêmes formes de la domination et de la subordination, de la concurrence, de l'imitation, de l'opposition, de la division du travail, nous trouvons la formation d'une hiérarchie, l'incarnation des principes directeurs des groupes en symboles, la division en partis, nous trouvons tous les stades de la liberté ou de la dépendance de l'individu à l'égard du groupe, l'entrecroisement et la superposition des groupes mêmes, et certaines formes déterminées de leur réaction contre les influences extérieures. » (Simmel, ib., nous soulignons)
\end{abstract}

Ces « influences extérieures » font écho au projet de la sociologie de l'environnement. Celle-ci cherche en effet à intégrer les phénomènes matériels, naturels, environnementaux dans l'approche sociologique afin de participer à l'analyse des causes et des conséquences sociales des phénomènes écologiques et des causes et conséquences écologiques des phénomènes sociaux. Si la thématique environnementale n'avait pas lieu d'être lors de la naissance de la sociologie, à l'époque de Simmel donc, il n'en reste pas moins vrai que les sociologues du tournant du XIXème siècle ont souvent, à un moment donné, abordé des questions proches de celles qui concernent aujourd'hui l'environnement, soit dans leurs discussions des connexions entre les réalités sociales et naturelles, soit dans l'étude plus directe des rapports des sociétés avec leurs milieux naturels.

Notre exposé se fera en trois temps. Avant toute chose, pour s'assurer de la légitimité des fondements de notre entreprise, nous dirons quelques mots, dans le prolongement de cette introduction, sur la pertinence de considérer Simmel comme un classique et de faire de l'environnement une question phare de notre époque. Ce n'est qu'ensuite que nous en viendrons au fait : de quelle manière Simmel parle-t-il de l'environnement en tant qu'interaction nature-société ? Il est clair que la définition de l'environnement comme interaction entre phénomènes naturels et phénomènes sociaux ne peut que faire écho à la vision simmelienne de la sociologie. Cependant, nous montrerons qu’il a développé une réflexion plus générale sur les relations entre logiques naturelles et logiques sociales qui englobe également ses propos sur la forme, la spatialité et la temporalité des phénomènes sociaux. Ceci permettra de compléter les recherches de Gross qui soulignent la capacité productive des possibilités préfigurées dans les éléments naturels.

On ne saurait toutefois être complet si l'on n'abordait pas la place de Simmel dans le récit de construction de la sociologie de l'environnement. Il ne s'agit pas de limiter l'apport du sociologue allemand

Émulations - Revue des jeunes chercheurs en sciences sociales 
au seul domaine de l'analyse sociologique de l'environnement : Simmel est un de ces sociologues dont la contribution ne peut être circonscrite dans un cadre limité. Par contre, chaque courant et domaine sociologique a besoin de recourir à des pères fondateurs ${ }^{2}$. Parce que la sociologie de l'environnement est un domaine encore récent et parce que son propos et encore parfois trop mal compris, elle cherche sans doute plus que d'autres domaines à s'intégrer dans l'histoire de sa discipline générale. Ainsi, bien que la sociologie allemande soit marquée par un certain isolement en cette fin de XIXème siècle - qui plus est si on la compare à son homologue français - elle n'en demeure pas moins inscrite dans les débats de son époque et influente : nous montrerons d'une part que la pensée de Simmel en général et sa sociologie de la forme en particulier doivent être rapprochées du courant de morphologie sociale ; d'autre part que l'influence de Simmel sur Park et l'écologie humaine participe elle aussi de l'inscription de Simmel dans les fondements de l'approche sociologique de l'environnement et par là de la sociologie de l'environnement dans la sociologie lato sensu.

En suivant ce projet qui fait de Simmel à la fois un penseur de l'interaction nature-société comme objet sociologique et un acteur de l'inscription sociologique de l'étude de l'environnement, la conclusion de cet article rappellera alors combien la sociologie de l'environnement et la science en général ne sont que des institutionnalisations d'activités cognitives

\subsection{L'environnement, une question sociale}

Examinons tout d'abord la pertinence de lier la pensée de Simmel à la «question de l'environnement» et d'associer ce projet dans la relecture des « classiques ». Il apparaît que la véritable socialisation de la nature qui s'est accélérée dans la seconde moitié du XX ème siècle a révélé les interconnexions entre les activités humaines et les logiques écologiques, faisant de l'environnement une thématique phare des enjeux scientifiques et politiques et l'entrainant au cœur des préoccupations contemporaines. Et réciproquement, depuis que l'environnement est entré sur la scène politique, médiatique, civile et économique, c'est-à-dire dans la sphère sociale, il est devenu, nécessairement, un objet d'étude pour les sociologues.

Jusqu'à présent, les sociétés occidentales et leur tradition scientifique, inspirée de la science galiléenne et newtonienne, ont presque toujours séparé les deux éléments, la société et la nature, pour les étudier chacun indépendamment de l'autre, «introdui[sant] un abîme entre le domaine de l'action humaine et celui des lois de la nature» (Bourg, 1993 : 7). La récente prise de conscience des dommages causés par certaines activités humaines sur ce milieu naturel a eu pour conséquence de mettre en question cette séparation, en la présentant comme une construction sociale voire en l'abolissant totalement. Il apparait qu'une telle reconnaissance des "problèmes » d'environnement a d'abord été impulsée par les sciences naturelles, mais bien des penseurs ont su associer ces préoccupations environnementalistes à des thématiques sociales, comme la technique, la population, les systèmes scolaires et médicaux et plus généralement aux limites d'une civilisation industrielle, de son système de pouvoir, de ses technosciences, en un mot d'une société et d'une humanité de plus en plus inhumaine et mécaniste (Duclos, 2006 : 9 ; Juan, 2007). Cette association des préoccupations environnementales et des questionnements sociaux pourrait d'ailleurs définir l'écologie politique; elle s'impose en tous cas comme l'une des composantes majeures de ce changement de millénaire : «il apparaît que notre question sociale en cette fin de siècle et au siècle suivant sera la question naturelle » (Moscovici : 2002 : 23).

Bien entendu, comme la plupart des universaux anthropologiques l'environnement est apparu avec l'humanité elle-même : les sociétés passées ont toutes eu affaire à des problèmes d'ordre écologique.

2. Et moins souvent, il est vrai, à des mères... La sociologie japonaise de l'environnement considère toutefois que N. Iijima est la mère de ce domaine avec son article de 1970 sur les incidences de la pollution de la baie de Minamata (cf. Iijima, 1970), par ailleurs la sociologie francophone est assez largement féminine aujourd'hui.

Émulations - Revue des jeunes chercheurs en sciences sociales 
Quelques exemples sont proposés par Broswimmer ([2002] 2003), à propos de la surexploitation de bois dans la Grèce antique ou sur la mythique île de Pâques, ou plus récemment concernant l'amenuisement des populations de bisons polonais durant la seconde Guerre Mondiale. Ce sont des exemples de destructions humaines de la nature. D'autres exemples sont encore plus célèbres, mettant cette fois la nature dans le rôle de la force cruelle : du mythique engloutissement de l'Atlantide par les eaux, à la plus tangible destruction de la vie humaine par les cendres du Vésuve ou encore le séisme de 1755 mis en vers par Voltaire (1756). Aujourd'hui plus que jamais, parce que l'environnement est devenu une menace causée par l'interaction des activités humaines et des forces naturelles, «la situation semble - à moins que notre intérêt propre ne nous aveugle - s'être dramatiquement détériorée » (Luhmann, 1993 : 77).

Depuis la fin des années 1960 de nombreux évènements marquants ont été organisés pour signaler l'urgence pour l'humanité de se consacrer à la question de l'environnement : différents ouvrages dont celui de Carson ([1962] 1963) ou le rapport Meadows (1972) puis celui de Brundtland (CMED, 1987), différents sommets mondiaux organisés par les Nations Unies, divers protocoles comme ceux de Montréal ou de Kyoto, sans oublier la montée des parties écologistes depuis la candidature de Dumont en France en 1974 aux innombrables associations de défense de l'environnement : tout cela est bien la preuve que l'environnement est désormais une préoccupation sociale majeure, sinon la seule question urgente à traiter.

\subsection{Simmel, un sociologue classique}

Faut-il maintenant légitimer le qualificatif de classique et son association au nom de Simmel ? Quelques considérations suffiront à convaincre du caractère classique de la sociologie de Simmel. On peut d'abord signifier son inscription dans la période des classiques puisqu'il naît la même année que Durkheim et meurt un an après lui. Il a surtout écrit de nombreux ouvrages de sociologie ou pour le moins intitulés «sociologie » comme sa Soziologie de 1908 (Simmel, [1908] 1999), et d'aussi nombreux articles dont celui paru dans le premier tome de L'Année Sociologique (Simmel, 1897) qui inaugure, avec Durkheim (1897), la revue fondatrice de la sociologie française, ou encore ceux rassemblés dans le célèbre recueil Sociologie et Epistémologie (Simmel, 1981). Il a été, avec Weber et Tönnies, un des fondateurs de la Société Allemande de Sociologie, mais aussi un des membres de l'Institut International de Sociologie initié par Worms. Enfin, et surtout, Simmel est un sociologue classique parce que son œuvre est toujours étudiée et enseignée aujourd'hui. Les ouvrages de Léger (1989), Vandenberghe (2000) ou Watier (2003) mentionnés plus haut réaffirment l'intérêt actuel pour ce sociologue - intérêt que ce numéro de la revue Emulations ne dément pas.

Ce qui a freiné la reconnaissance de la sociologie de Simmel, c'est peut-être le fait que Simmel ne se considérait pas lui-même comme un sociologue, comme il l'écrit dans une lettre à Bouglé :

«D’une manière générale, je suis quelque peu affligé qu'on me considère seulement, à l'étranger, comme un sociologue, alors que je suis en fait un philosophe, que je tiens la philosophie pour la tâche de mon existence et ne pratique au fond la sociologie que comme domaine accessoire. » (Lettre à C. Bouglé, cité par Vandenberghe, $2000: 2$ )

Watier (2003 : 9) nuance ces propos de Simmel en adoptant un autre angle d'approche : «Il a écrit une Sociologie de plus de 700 pages mais n'a jamais considéré, sur le modèle comtien, qu'elle serait la reine des sciences, ni qu'elle seule serait susceptible de couronner l'édifice scientifique ». C'est sans doute pour ce manque d'enthousiasme sociologique que l'école Durkheimienne en particulier et les sociologues dans leur ensemble ne lui accorderont qu'une reconnaissance mitigée et surtout tardive, et peut-être également à cause de ses références à la psychologie, qui s'entr'aperçoivent dans ses accents bergsoniens, ou encore dans la tonalité compréhensive et individualiste visible cette fois dans ses liens avec Weber.

Émulations - Revue des jeunes chercheurs en sciences sociales 
Quoiqu'il en soit, la légitimité d'étudier Simmel dans son analyse de l'environnement, une fois accepté que Simmel est un classique et l'environnement une question incontournable, tient alors dans le raisonnement suivant : en plus de la tradition sociologique de relecture des classiques, étant données les difficultés qu'ont rencontrées les sociologues intéressés par la thématique de l'environnement pour «faire » domaine de recherche sociologique, toute entreprise de légitimation supplémentaire est bienvenue pour ce champs. Après des tentatives d'ordre révolutionnaire (e.g. Catton \& Dunlap, 1978 et le commentaire Buttel, 1978), les sociologues de l'environnement ont choisi d'inscrire l'environnement dans le domaine de la sociologie par des voies plus «classiques », dont la démonstration que déjà les classiques avaient, peu ou prou, abordés d'un point de vue sociologique le rapport à la nature des sociétés (Boudes, 2008). C'est à chaque fois un pari risqué pour la sociologie de l'environnement que de tenter une telle justification, mais il semble que la plupart des tentatives aient réussi, du moins celles mentionnées plus haut ${ }^{3}$.

\section{Simmel et la question de l'environnement : forme, nature, espace}

\subsection{Une sociologie de la forme}

Simmel s'est intéressé à de nombreux aspects de la vie sociale, dont les plus anodins. Comme le rappel Watier (2003 : 14) : «Il ébauche une sociologie des sens, de la vue, de l'ouie [...] et insiste sur des catégories psychosociales, des 'états psychiques' tels que la fidélité, la confiance, la reconnaissance, la piété, qui entrent comme matière ou contenu dans les socialisations, voire les supportent » (nous soulignons). Ce que cherche Simmel c'est bien la matière de la socialisation, l'expérience physique de l'interaction et les formes des relations sociales. Il propose une sociologie de la forme qui vise à placer un outil d'interprétation intermédiaire entre l'individu et la relation sociale. Il s'agit de se désintéresser du contenu pour mieux se tourner vers le contenant, se détacher du fond de la relation sociale pour appréhender sa forme.

Cet aspect de la sociologie de Simmel qui «étudie les formes d'associations qui font la société en structurant les interactions entre individus » (Vandenberghe, $2000: 37$ ) nous intéressera ici particulièrement ${ }^{4}$.

«C'est sur cette [dernière] considération abstraite des formes sociales, précisait Simmel dès 1897, que repose tout le droit que la sociologie a d'exister. [...] Les formes qu'affectent les groupes d'hommes unis pour vivre les uns à coté des autres, ou les uns pour les autres, voilà le domaine de la sociologie. » (Simmel, $1897: 172)$

\footnotetext{
${ }^{3}$. En poursuivant le travail de Gross montrant l'intérêt des travaux simmeliens sur l'interaction entre nature et société, on prend ici peu de risques. D'autres tentatives furent moins heureuses : il est par exemple relativement difficile de prétendre que M. Halbwachs acceptait de prendre en compte l'action des phénomènes naturels sur les sociétés, malgré son attachement à la morphologie sociale. Nous avions espéré valider une telle hypothèse lors d'un colloque sur Halbwachs (Boudes, 2007), mais des spécialistes de ce dernier on fait de notre tentative un échec - pour la sociologie de l'environnement s'entend, mais une réussite en tant que contribution à l'histoire de la sociologie.

4. Vandenberghe explique que si pour Simmel « tous les domaine de la vie trouvent leur origine et leur fondement dans les interactions entre les individus » (2000:36), ses travaux peuvent dès lors être découpés en trois catégories. Une sociologie générale et macro-historique où l'on retrouve la plupart des accents bergsoniens, une sociologie philosophique qui inclut ses apports épistémologiques et projette " une vision métaphysique de la vie sociale » (ib. : 37), enfin une sociologie formelle ou microsociologie ahistorique qui «étudie les formes d'associations qui font la société en structurant les interactions entre individus » (ib.: 37).
}

Émulations - Revue des jeunes chercheurs en sciences sociales 
Cependant, bien qu'une large part de l'œuvre simmelienne se retrouve associée à une forme d'interactionnisme, seule une partie minime aborde l'aspect matériel de ces interactions. C'est dans sa Soziologie (Simmel, [1908] 1999) qu'un chapitre sur l'espace traite de cet aspect. Comme le rapporte Watier (2003: $59-60)$ :

«[Ce chapitre] s'intéresse à la manière dont les formes de socialisation réalisent une forme spatiale,
construisent les distances et les proximités, les frontières et les ponts, qui marquent sa clôture. [...]
[Simmel se donne pour but] d'éclairer les modes selon lesquels les formes de socialisation deviennent
réelles spatialement. [...] [L]'espace fonctionne comme contexte pour l'action et l'espace n'a de
forme que sociale, et cependant il n'est pas qu'une construction sociale (il se déploie en facteur social
et/ou physique en complément à d'autres facteurs). Il est donc possible d'analyser, d'une part, les
aspects de l'espace qui ont une importance sociologique, de l'autre l'effet des formes sociales sur
l'espace.»

Dans cette approche de l'interaction, l'essence de la société tient dans les unités sociales en tant qu'elles s'inscrivent spatialement et qu'elles prennent conscience de cette inscription dans le temps. Pour Simmel, la socialisation et l'interaction créent la société, à travers non seulement la rencontre des individus (au sens spatial) mais surtout la conscience de cette rencontre. C'est cette conscience qui fait de la rencontre une interaction, qui devient elle-même l'interaction, « ce qui différencie dès lors toute unité sociale de tout phénomène naturel, [car] pour ce dernier la coexistence spatiale ne s’y redouble pas instantanément de la conscience de cette coexistence» (ib.: 37). Simmel donne ainsi à l'espace une place majeure dans l'analyse sociale, par exemple dans la comparaison entre la forme sociale «Etatnation» et celle «société nomade»: la première se définit par rapport au territoire qu'il gouverne (fixation), la seconde par sa mobilité au sein d'une pluralité d'espaces qui sont pourtant les fondements de sa spécificité (mouvement).

\subsection{Une sociologie ouverte aux phénomènes naturels}

Cette première approche une fois définie, Simmel va progressivement s'ouvrir aux phénomènes naturels et tentera de concilier la nature et la société dans son concept d'interaction. Comme le rappelle Groos (2001 : 397), « le noyau de la notion de Wechselwirkung de Simmel peut être traduit par 'interaction réciproque' mais aussi, en référence à la causalité, par 'effet réciproque' 'et causation réciproque'». C’est dans ce sens que Gross affirme que la définition simmelienne de la société n'est pas exclusive : elle inclut au contraire l'ensemble des interactions entre humains mais aussi entre humains et non humains parce que ce dernier type d'interaction participe également des connections qui forment les sociétés.

Deux aspects de la réflexion de Simmel sont à distinguer ici. D’abord, son analyse consiste à différencier la nature culturalisée et la nature cultivée (Gross, 2001). Dans le premier cas, il s'agit de choses fabriquées par l'humain (buman-made things), dont la ville ou un mât de bateau que Simmel prend en exemple. Dans le second cas, il s'agit de nature fabriquée par l'humain (buman-made nature), comme un paysage ou un fruit issu d'un arbre cultivé. En effet, Simmel prend l'exemple d'un arbre fruitier: le fruit que donne un arbre cultivé demeure un élément naturel en tant qu'il est le résultat de la tendance de l'arbre à faire des fruits. Par contre, si à partir de cet arbre on fait un mât, le résultat ne peut pas être compris comme l'aboutissement d'une tendance naturelle des arbres à faire des mâts et il s'agit dès lors de nature culturalisée.

Pour Gross, cela signifie que toute production humaine matérielle, selon qu'elle suit les tendances de la nature ou qu'elle les transforme, fabrique à nouveau de la nature ou au contraire de la culture :

«Pour parler d'un point de vue contemporain, commente Gross (2001: 400), dans cette conception les dynamiques écologiques naturelles sont initiées par les humains, mais par la suite la nature, pour ainsi dire, se conçoit elle-même (designs itself). Cette 'auto-conception' ('self-design') peut entrainer de

Émulations - Revue des jeunes chercheurs en sciences sociales 
nombreux résultats potentiels en raison de la complexité et de l'imprédictibilité des dynamiques écologiques. »

C'est là toute la portée de l'interaction (Wechselwirkung) : la nature cultivée est le résultat d'actions humaines mais demeure indépendante en tant qu'elle contient un développement propre à elle et latent, et qu'elle peut agir en retour sur l'évolution des productions humaines.

Ceci nous conduit au second point, lorsque que Simmel propose l'exemple significatif d'une ruine. A travers cet exemple, explique Gross (ib.) :

«Simmel souligne que, bien qu'une ruine fut à l'origine une œuvre architecturale, les deux formes de l'environnement matériel - un arbre cultivé aussi bien qu'une construction culturelle - peuvent développer leur propre vie 'naturelle' et par là peuvent évoluer en quelque chose qui se transmute en un produit de nature, indistinguable pour les humains de la nature 'inviolée' ('untouched'). ».

En quelque sorte donc, quand bien même une ruine est d'abord une œuvre humaine, la nature s'approprie littéralement cette production délaissée pour en faire une œuvre cultivée - et non plus culturelle ou culturalisée. La ruine devient dès lors « le point de rencontre ou l'interface de l'action humaine et de l'action nature » (Gross, ib. : 403). Qui plus est, par cette action la nature interagit bel et bien avec les phénomènes culturels, ce qui permet à Simmel de généraliser : "la nature a transformé l'œuvre d'art en matériel pour sa propre expression » (Simmel, 1986 : 120, cité par Gross, 2001 : 400). En somme, la distinction entre nature cultivée et culturalisée prend tout son sens une fois associée au concept d'interaction et permet de réaffirmer, aujourd'hui, la complexité des phénomènes d'environnement en tant qu'ils résultent de logiques sociales et naturelles indépendantes en ellesmêmes mais connectées dans les faits.

\title{
2.3. Une sociologie de l'espace
}

Enfin, Simmel propose également une analyse spatiale des phénomènes sociaux dont ont doit retenir comme exemple sa célèbre digression sur l'étranger dans le chapitre sur l'espace de sa Soziologie. Celle-ci est souvent considérée pour elle-même et amenuise la portée de cette ébauche de sociologie de l'espace. Néanmoins, si on le replace dans le contexte du chapitre, comme s'y emploie Vandenberghe, on peut en retenir ceci :

\begin{abstract}
«Simmel présente une analyse 'constructiviste' de la détermination spatiale de la société en examinant, de façon symétrique, la construction spatiale du social (comment les formes spatiales, telles que les frontières, la proximité ou les mouvements migratoires, structurent-elles les interactions sociales), et la construction sociale du spatial (comment les interaction sociales s'expriment-elles symboliquement dans les formes spatiales? - par exemple : le terrain vide comme expression de la neutralité). » (Vandenberghe, $2000: 67$ )
\end{abstract}

Dit autrement, Simmel nous éclaire directement sur les rapports des sociétés avec leur milieu : le propre de la société, selon lui, serait d'accorder certes une importance davantage symbolique que géographique ou physique au territoire, mais c'est à travers cette construction de l'espace social - de l'espace de la société - que l'on peut rendre compte des phénomènes sociaux et comparer les degrés d'importance symbolique donnée au territoire.

Il est donc possible d'exprimer une forme sociale par rapport à l'espace et, réciproquement, de rendre compte d'une configuration spatiale par sa construction sociale. D'ailleurs, cette digression sur l'étranger doit être considérée comme un exemple d'analyse spatiale. L'étranger est d'abord perçu par la société hôte comme une incursion dans sa zone socio-spatiale avant d'être vécu comme une incursion socio-culturelle. Et Simmel ([1908] 1979 : 59) ne définit-il pas la «forme sociologique» de l'étranger comme l'union tout à la fois de l'errance et du mouvement avec la fixité et l'inscription spa-

Émulations - Revue des jeunes chercheurs en sciences sociales 
tiale ? Il ajoute même plus loin (ib.) que c'est bien la combinaison particulière des dimensions de distance et de proximité qui produit la relation spécifique et formelle à l'étranger. Si ces propos sont généralement minimisés par les commentateurs de Simmel (pas plus de trois pages chez Vandenberghe, cinq ou six chez Watier), c'est qu'ils entrent directement en résonance avec les caractéristiques des stratégies individuelles d'autorégulation des relations interpersonnelles. Simmel insiste en effet sur la fonction d'une forme originelle de retenue, le tact et la réserve, qui serait la forme pure de l'interaction. Prenant acte du fait que, dans les grandes villes, les relations ne peuvent être que superficielles étant donné leur nombre (sinon elles causeraient purement et simplement un surmenage mental produit par intensification de l'activité nerveuse développée), Simmel rend compte des interactions en tant que relations artificielles. D’un coté elles représentent un besoin universel de socialisation et de sociabilité, d'être ensemble, de l'autre elles ne peuvent être que des artifices de relations vides de tout contenu que seuls le tact et la réserve permettent de perpétuer (cf. Grafmeyer \& Joseph, 1979 : 47-48).

C'est en tous cas ce désintérêt pour le contenu, pour le fond de la relation, qui permet le mieux de comprendre cet attachement à la forme. C'est aussi pour cela que l'étude de l'inscription spatiale des interactions se limite à des termes abstraits et non à une mise en lien directe des sociétés avec leur milieu physique : Simmel n'accorde pas d'importance à la matérialité de ce rapport à l'espace - et c'est en cela qu'il se distingue clairement d'une approche géographique - mais bien à ses constructions sociales et à ses implications dans les relations. Et dans le même temps, il ne peut se contenter de mettre sur le même plan les fabrications humaines artificielles et celles liées à la nature. Il distingue une sphère strictement sociale, celle qui permettra à la sociologie de trouver sa place sur l'échiquier des sciences, et qui est constituée par l'ensemble des interactions inter-humaines. Toutefois celles-ci sont incluses dans un ensemble plus général : non seulement elles ont des formes matérielles et s'inscrivent spatialement, mais en plus elles appartiennent à l'ensemble des interactions en tant que phénomène primordial. Et s'il est possible de distinguer des interactions inter-humaines, il est également possible de délimiter des interactions entre phénomènes naturels; le contenant global est alors l'ensemble des interactions entre humains et/ou non-humains, de la même manière que la question de l'environnement englobe aujourd'hui l'ensemble des relations entre humains et/ou non-humains.

\section{La pensée de Simmel et les fondements de l'approche sociologique de l'environnement}

Ce qui fait de Simmel un cas particulier de l'étude des classiques dans leur traitement de l'environnement, c'est qu'en plus de sa contribution générale et théorique à l'approche sociologique des relations nature-société, il a influencé directement les courants pionniers en matière d'analyse sociologique de l'environnement. Si Durkheim, Weber ou Marx ont abordé peu ou prou les questions contemporaines de l'environnement, aucun n'a été suivi pour ces travaux-ci. Par contre, Simmel a notamment contribué à l'émergence de l'approche écologique en sociologie, et dans un autre registre il a indirectement participé à l'approche morphologique des phénomènes sociaux. Le prolongement de sa pensée est visible dans ces deux premiers courants de l'histoire de la sociologie de l'environnement: l'écologie humaine et la morphologie sociale. Comment ne pas voir dans la première la continuité avec la vision simmelienne de l'interaction et son intérêt particulier pour la spatialisation des phénomènes sociaux ? De même pour la seconde, il y a de nombreux points communs entre une morphologie sociale qui cherche à rendre compte des formes matérielles des phénomènes sociaux et la sociologie de la forme et de l'espace proposée par Simmel.

De plus, morphologie sociale et écologie humaine sont les deux courants qui ont su orienté la sociologie vers les phénomènes les plus matériels, les plus naturels et par là les moins strictement sociaux. Faire de Simmel, en quelque sorte, un entremetteur de ces deux courants permet de réaffirmer la cohérence, mais aussi la nécessité, de l'approche sociologique de l'environnement et, encore une fois, son inscription dans la sociologie générale dès la naissance de celle-ci. En revenant maintenant sur les influences du sociologue allemand sur ces courants étasunien et français, on ne se contente plus de re-

Émulations - Revue des jeunes chercheurs en sciences sociales 
lire, in abstracto, Simmel dans son approche sociologique de l'environnement, on voit se construire devant nous l'histoire même de la sociologie de l'environnement.

\subsection{Simmel, Park et l'écologie humaine}

C'est principalement Robert E. Park qui transposera les préceptes simmeliens à l'analyse empirique de la ville. Ici réside tout l'intérêt porté à Simmel car à la différence des autres classiques, dont les éléments d'analyse de l'interaction nature-société n'ont pas été prolongés, les idées de Simmel vont être directement reprises, de l'autre coté de l'atlantique, via Park, figure éminente de l'école de Chicago. Aux réflexions sur les formes des relations sociales en lien avec la spatio-temporalité des interactions vont s'ajouter les apports de l'écologie naturaliste pour ériger une des approches phares de l'école de Chicago : l'écologie humaine, sur laquelle s'appuieront les premiers sociologues de l'environnement pour élaborer leur domaine et asseoir la légitimité de l'approche sociologique de l'environnement (cf. e.g. l'article de Buttel, 1986, au titre évocateur : La sociologie de l'environnement : la lente maturation de l'écologie humaine)

Revenant d'un séjour en Europe, Park disait toujours combien son approche était redevable à Simmel dont il tient sa « seule instruction formelle en sociologie » (Park, 1950 : vi, cité par Grafmeyer \& Joseph, 1979 : 44). Vers 1897, après avoir commencé une carrière de journaliste, il reprend des études de psychologie sociale et effectue un séjour de trois années en Allemagne où il est l'élève de Simmel à Berlin, puis à Strasbourg où celui-ci sera muté. La sociologie étasunienne à l'époque de Park subit un fort développement institutionnel et théorique. Un développement institutionnel lié à une demande croissante d'analyse des transformations de la société américaine. Une évolution théorique et pratique engendrée par le renouvellement de questions sociologiques toujours plus proches de l'individu et de ses rôles et fonctions sociaux dans la modernité, ce qui peut expliquer l'engouement que rencontre Simmel.

Quelques temps après son retour d'Europe, Park se fera recruter par Thomas et tous deux demeurent, avec Small, Znaniecki, McKenzie, Wirth et Burgess les figures représentatives de la fondation de l'école de Chicago (Alihan, [1938] 1964 ; Grafmeyer \& Joseph, 1979). Toutefois, c'est Park et Burgess, durant les années 1920 et 1930 qui reprirent les réflexions simmeliennes. «Dans leur manuel de sociologie, explique Watier (2003 : 134), Burgess et Park insistent sur l'interaction sociale telle que la définit Simmel : elle caractérise le groupe dans le temps et l'espace ». La sociologie urbaine de Chicago est le prolongement des interrogations de Simmel sur les relations des individus au sein des grandes villes et sur l'implication spatiale des interactions.

Un exemple probant de la reprise des idées de Simmel est celui de l'explication donnée par Park des phénomènes d'intégration de la population noire. Il faut d'abord rappeler qu'entre 1903 et 1913 Park est également l'assistant du leader noir Booker Washington. C'est dans ce contexte que le sociologue s’interroge sur les phénomènes de colonisation, principalement au Congo où celle-ci est essentiellement du ressort des grandes compagnies coloniales. Ce n'est alors pas surprenant si « $[\mathrm{u}] \mathrm{n}$ de ses thèmes majeurs est l'assimilation des jeunes Noirs du sud des Etats-Unis ; [pour Park] cette assimilation doit se faire par l'éducation et la formation professionnelle. » (Cuche, $2000: 7-8$ )

Le problème que pose, à l'époque, la population noire aux blancs relève d'un questionnement relatif aux migrations et à l'intégration sociale. Les populations noires ont servi de bouc émissaire au problème de migration; mais la position de Park va être de dire qu'il est dans la logique de la société d'assimiler des étrangers/migrants, que c'est la logique «naturelle » de la société «qui conduit inéluctablement à l'intégration » $(i b .: 8)^{5}$.

\footnotetext{
5. Cf. Cuche, 2000, où l'auteur montre très utilement le processus d'assimilation des migrants par la société d'accueil selon Park. Quatre étapes successives définissent ce processus :
}

Émulations - Revue des jeunes chercheurs en sciences sociales 
D’après Park, les sociétés assimilent naturellement des étrangers ou migrants. L'analyse qu'il propose applique tout à fait le principe simmelien selon lequel « [s]i la mobilité s'introduit dans un groupe fermé, elle entraîne avec elle cette synthèse de proximité et de distance qui constitue la position formelle de l'étranger.» (Simmel, [1908] 1979 : 54). D'ailleurs, lorsque Park systématise ses travaux, il reprend les expressions simmeliennes. Par exemple quand il introduit son article «La communauté urbaine, un modèle spatial et un ordre moral »-dont le titre est déjà probant - il explique que :

« Si, en société, nous vivons ensemble, nous vivons aussi en même temps à l'écart les uns des autres, de sorte que les relations humaines peuvent toujours être analysées, avec plus ou moins d'exactitude, en terme de distance. Dans la mesure où une structure sociale peut se définir en terme de positions, les changements sociaux peuvent être décrits en terme de déplacements. » (Park, [1926], 1979 : 194)

Ou encore, dans le même article (ib.: 202) :

«La mobilité mesure le changement social et la désorganisation sociale, parce qu'un changement social entraîne toujours un changement de position dans l'espace et que tout changement social, même celui que nous décrivons comme progrès, entraîne une désorganisation sociale. »

Ce sont de tels rapprochements, et d'autres encore, qui poussent Grafmeyer \& Joseph (1979 : 43) à écrire que Park et Simmel sont les premiers théoriciens de la distance sociale et de son principe de socialité, le principe de réserve. Il est dès lors surprenant que peu de textes pointent ce lien entre Simmel et Park. Le travail de synthèse sur l'écologie que réalise Alihan ([1938] 1964), tout en présentant Park comme le fondateur de ce courant ne cite Simmel qu'une seule fois, à propos du voyage de Park en Allemagne ${ }^{6}$. La publication de la Human ecology de Hawley (1950) éviterait également le rapprochement avec Simmel et aurait, par ailleurs, définitivement « enracin[é] l'écologie humaine dans la morphologie sociale de Durkheim» (Buttel, 1986 : 364). Un article de Schnore (1958) poursuivra cependant cette analyse des liens entre les deux courants en révélant les nombreuses similitudes entre les deux approches - similitudes à réaffirmer aujourd'hui alors même que d'aucun mettent en avant la seule inspiration naturaliste de l'écologie humaine négligeant ainsi ses soubassements simmeliens et morphologiques (e.g. Hannigan, $2006: 16-18)^{7}$.

1) Compétition : Chaque fois que des immigrants arrivent, il y a compétition entre les nouveaux venus et les anciens occupants. A ce stade, il n'y a pas de contact entre les deux groupes. La compétition relève d'un processus inconscient.

2) Conflit. Il faut que le conflit soit ouvert et conscient. Le conflit est normal parce que structurant : il structure les relations entre les groupes en présence et développe des structures de solidarité. Le conflit est un progrès par rapport à la compétition. Il permet au groupe dominé d'organiser sa réaction. Le conflit est donc positif.

3) Accommodation. Il s'agit de s'adapter et de s'ajuster aux nouvelles conditions ayant émergé du conflit. Celui-ci n'est plus ouvert, ce qui n'enlève rien à la conflictualité. L'accommodation peut déboucher sur un nouveau conflit ou sur l'assimilation. Cette phase renvoie à une réorganisation sociale.

4) Assimilation. Park propose une théorie des relations entre groupes sociaux qu'il a publiée dans son Introduction to the science of sociology (Park \& Burgess, 1921). La finalité du processus débouche sur l'assimilation des migrants dans la société d'accueil, à travers un compromis culturel. L'assimilation n'est pas la conversion à la culture du groupe dominant ; c'est un compromis culturel qui permet aux groupes de conserver leur culture recomposée tout en participant à la culture de la société d'accueil. Cela suppose un processus d'interaction. L'assimilation constitue un processus d'interpénétration et de fusion par lequel on partage l'expérience, l'histoire mais aussi l'espace pour avoir une vie culturelle commune.

6. «A Berlin, Strasbourg et Heidelberg, [Park] travailla avec Simmel et Windelbend, rédigeant sa thèse sous la direction de ce dernier. » Alihan, [1938] $1964: 4$

7. Cependant, l'exclusion de la référence à Simmel dans la présentation de l'écologie humaine est compréhensible - surtout pour des ouvrages des années 1930 ou 1950. D'abord, c'est Durkheim qui est le sociologue influent. S'il a été dit plus haut que Simmel est aujourd'hui un classique comme les autres, Durkheim parait être le nom reconnu à l'époque de l'école de Chicago. Ensuite, il existe une forte homologie entre les emprunts de l'école de Chicago à l'écologie et ceux de Durkheim à la théorie de l'évolution (Boudes, 2008, chap. III). Enfin, bien que Simmel demeure indéniablement un inspirateur de Park, c'est davantage le modèle de l'écologie naturaliste qui est mis en

Émulations - Revue des jeunes chercheurs en sciences sociales 


\subsection{Simmel, Halbwachs et la morphologie sociale}

Si l'écologie humaine et la morphologie sociale sont souvent associées pour servir une même ambition, cela invite à y voir encore la trace, indirecte mais certaine, de Simmel. De même que nous avons lié Simmel à Park et à l'écologie humaine, nous pouvons le rapprocher de Halbwachs voire de l'entière morphologie sociale, c'est-à-dire du second courant fondateur de la sociologie de l'environnement avec l'écologie humaine. Deux aspects majeurs sont à considérer dans la comparaison entre Halbwachs et Simmel : leur attachement à une sociologie psychologique inspirée notamment de leurs lectures reconnaissantes de Bergson, et leur prise de position pour une analyse des formes de la société (morphologie sociale et sociologie des formes).

Dans le premier cas, on peut rappeler que Simmel portait son attention autant sur les interactions physiques que sur la conscience humaine de ces interactions. Or cela apparaît très proche de ce que disait Halbwachs dans sa Morphologie sociale:

«Il y a donc bien là tout un ordre de représentations collectives qui résultent simplement de ce que la société prend conscience, directement, des formes de son corps matériel, de sa structure, de sa place et de ses déplacements dans l'espace, et des forces biologiques auxquels il est soumis.» (Halbwachs, [1938] 1946 : 191)

Cela s'observe également chez Simmel qui déploie une sociologie psychologique dans laquelle ce sont bien les interactions individuelles qui fabriquent la société en prenant conscience de leurs actes ${ }^{8}$.

Les deux sociologues, Halbwachs et Simmel, souhaitaient redonner une apparence matérielle aux symboles sociaux. Après tout, la distribution au sol de la population, objet cher à la morphologie sociale, peut être considérée comme un fait purement physique, tout comme les formes matérielles des interactions. Halbwachs va d'ailleurs élaborer sa pensée en montrant que les formes matérielles ou naturelles de la société agissent non pas comme une contrainte, mais par la conscience que nous en prenons, c'est-à-dire par la perception psychique et la construction sociale que nous en faisons. De même, pour Simmel, l'association et la relation sociale se définissent par leur spatialité et par leur forme, par la conscience de la coexistence dans cette interaction. « Il y a là un genre de pensée ou de perception collective qu'on pourrait appeler une donnée immédiate de la conscience sociale qui tranche sur toutes les autres, et qui n'a pas encore été aperçue suffisamment par les sociologues eux-mêmes .» La phrase est de Halbwachs ([1938] 1946 : 191), mais elle irait tout aussi bien à Simmel, et l'on sent en tous cas cet accent bergsonien cher au sociologue allemand tant ces « données immédiates de la conscience collective » font écho au titre de l'ouvrage Essai sur les données immédiates de la conscience (Bergson, [1888] 2001).

Finalement, les limites entre sociologie et psychologie sont atteintes ici et ce sont d'ailleurs ces rapprochements avec la psychologie que Durkheim reprochera à Simmel puisqu'ils nuisent à la reconnaissance de la discipline sociologique comme science autonome. Ce sont pourtant ces mêmes idées que l'on retrouve en filigrane chez Halbwachs à tel point que la préface de l'édition de 1962 de sa Morphologie sociale laisse penser que «métaphysique et sociologie ont convergé chez Halbwachs vers une psychologie sociale enveloppante et fine » (Girard, 1962).

Dans un autre registre, un récent projet visant à lier morphologie sociale et morphologie spatiale s'appuie sur un parallèle semblable à celui que nous exposons.

avant pour caractériser l'ensemble des écrits de ce dernier et de ses collègues, ce qui fait passer l'influence du sociologue allemand au moins au second plan.

${ }^{8}$. D’où les reproches de Durkheim $(1900: 7):$ «Or de quel droit sépare-t-on si radicalement le contenant du contenu de la société. On affirme que seul le contenant est de nature social, et que le contenu ne possède qu'indirectement ce caractère. Cependant, il n'y a aucune preuve pour confirmer une assertion qui, loin de passer pour un axiome évident, peut surprendre le chercheur. » Et le même Durkheim d'ajouter plus loin (ib.: 8) : «Ce serait une idée tout à fait étrange que d'imaginer le groupe comme une espèce de forme vide, de moule indifférencié pouvant recevoir n’importe quel matière !»

Émulations - Revue des jeunes chercheurs en sciences sociales 


\begin{abstract}
«La morphologie sociale telle qu'elle a été définie par E. Durkheim, M. Halbwachs ou M. Mauss et la géométrie des formes sociale de G. Simmel et de ses disciples de l'école de Chicago (Park, Burgess), nous semblent constituer un substrat particulièrement fécond pour la tentative de rapprochement interdisciplinaire que nous proposons, dans la mesure où elle définit un objet de recherche relativement précis (l'influence de la structure du peuplement sur les conditions générales de la vie en société) qui intéresse a priori l'ensemble des sciences sociales, sans exclusive. [...] Et c'est bien sur le problème de la signification sociale des formes spatiales et de l'emboitement des différents niveaux d'organisation de la réalité sociale que nous pensons que le dialogue interdisciplinaire peut s'engager de la façon la plus fructueuse. » (Grasland, 1999 : 1-2)
\end{abstract}

Il est clair que la morphologie sociale, surtout dans ses développement halbwachsiens, n'a finalement pas donné suite à son intérêt originel pour le rapport au sol des sociétés (Boudes, 2007). Mais cela n'empêche pas de voir les homologies fondamentales entre morphologie sociale et sociologie des formes simmelienne. Dans l'idée de Grasland comme dans notre démonstration, l'école française est perçue comme complémentaire de celle de Chicago et de son inspiration simmelienne. Cette complémentarité apparaît également dans l'inclusion de l'article de Halbwachs ([1932] 1979) dans le recueil de textes sur l'école de Chicago établi par Grafmeyer \& Joseph (1979), article qui clôture une compilation introduite par deux textes de Simmel ${ }^{9}$. Enfin, Schnore (1959: 631) est sans doute un des premiers à voir cette homologie en faisant remarquer que la morphologie sociale de Halbwachs incorpore une perspective écologique «rigoureusement similaire à celle de Park », et en présentant le voyage du sociologue français aux Etats-Unis comme une preuve de cette complémentarité des approches.

\title{
Conclusion
}

Il est ainsi tout à fait raisonnable de dire qu'en plus des réflexions personnelles de Simmel sur les interactions entre phénomènes sociaux, spatiaux et naturels qui caractérisent aujourd'hui l'environnement, son influence sur les premiers courants sociologiques cherchant à intégrer des perspectives écologiques et/ ou morphologiques est certaine. Alors que la sociologie de l'après Seconde Guerre Mondiale ciblera les aspects strictement sociaux des interactions sociales, seule l'écologie humaine, en s'appuyant sur l'apport théorique de la morphologie sociale, continuera d'interroger les liens des sociétés avec leur bases matérielles. Il ne fait aucun doute qu'une raison sous-jacente à cette entreprise, pourtant à contre-courant de la sociologie dominante, était la conviction que, indépendamment des causes - ou du contenu - de l'action de la nature, l'influence de Simmel conduisait à interroger les manières d'agir de la nature - les formes de son action. En acceptant de se demander comment la nature agit ou réagit aux phénomènes humains et/ou sociosystémiques, les sociologues peuvent ainsi accéder à un questionnement sociologique plus fin concernant l'action humaine via la prise en compte d'une éventuelle action naturelle primordiale.

L'imprédictibilité de la nature ne prenait tout son sens, aux yeux de Simmel, qu'une fois confrontée à l'œuvre humaine - elle-même assez volatile. La séparation des phénomènes pour leur compréhension ultime ne convenait pas à Simmel, et il trouva dans l'analyse spatiale des phénomènes sociaux, et plus précisément dans l'étude du phénomène urbain, un champ de recherche à partir duquel ses idées prenaient sens. Prise en compte des interactions socio-naturelles et analyse de la ville ont pu converger vers le dévoilement d'une forme de complexité qui caractérise aujourd'hui les questions

9. A propos de cet article de Halbwachs publié par Grafmeyer \& Joseph, Topalov (2006 : 563) note que le lien entre l'école de Chicago est le sociologue est peu explicité. Topalov ne tient compte, cependant, que du commentaire de Grafmeyer \& Joseph associant l'approche morphologique à celle écologique (Grafmeyer \& Joseph, 1979 : 35), mais non pas d'un autre commentaire des deux directeurs d'ouvrage (ib.: 25) mettant en avant les tentatives imagées de Halbwachs ([1932] 1979 : 324) pour rendre compte au mieux de l'apport de cette école.

Émulations - Revue des jeunes chercheurs en sciences sociales 
d'environnement et que Simmel attribuait, à sa manière, à la ville, tout en la comparant à un organisme dont l'ensemble dépasse nécessairement la somme des éléments.

«Tout comme un être humain ne prend pas fin avec les limites de son corps physique ou les effets personnelles (the personal belongings) avec lesquels il ou elle est immédiatement en relation, mais plutôt, avec la totalité des effets significatifs qui émanent temporellement et spatialement de lui ou d'elle, d'une manière semblable une ville existe seulement dans la totalité de ses effets, lesquels transcendent leur sphère immédiate. C’est le véritable contexte dans lequel son être est exprimé.» (Simmel, 1903 : 200, cité par Gross, $201: 401$ ).

De même, les question sociales doivent être incorporées dans le contexte spatial et temporel qui les transcende et que traduisent les phénomènes d'environnement. C’est en ce premier sens que Simmel participe clairement de la sociologie de l'environnement.

Dans un second sens, Simmel participe encore de la sociologie de l'environnement parce qu'en plus d'être un des auteurs classiques les plus significatifs pour justifier l'heuristique d'une approche sociologique de l'environnement, il valide l'inscription historique de ce domaine dans la sociologie générale. Parce qu'il permet d'aborder dans un même élan la morphologie sociale et l'écologie humaine, il confère à la sociologie de l'environnement des bases stables et légitimes.

Cette «double» participation de Simmel à la sociologie de l'environnement, dans l'apport de connaissance et dans celui de légitimation, n’est pas sans rappeler les débats des sociologues des sciences sur la légitimité cognitive ou institutionnelle des sciences en générale ou d'un cas en particulier. Fort d'un premier travail sur ce point (Boudes, 2008 : chap. 13), nous pouvons réaffirmer ici la double inscription de la science en tant qu'institutionnalisation de l'activité cognitive ${ }^{10}$. Comme le rappelle justement Berthelot (2008) l'approche synchronique ou diachronique d'une science (ou d'un chercheur, d'un article, d'une démonstration) la fait percevoir davantage comme un phénomène social on cognitif ${ }^{11}$. Dans le cas de Simmel, il ne fait aucun doute que l'apport fut d'abord cognitif et que l'écologie humaine l'a en partie instrumentalisé ou, plus sûrement, a contribué à sa socialisation, tout comme les sociologues de l'environnement instrumentalisent parfois leurs prédécesseurs pour en faire des pionniers ou, au contraire, des ennemis privilégiés. Mais l'histoire passant, il semble qu'aucun des deux aspects ne puisse demeurer seul et que la science, pour faire science, doit toujours rassembler in fine ses caractéristiques cognitives et sociales.

\footnotetext{
10. Cf. également Berthelot, 2008 : 101-102 (chap. 5) où il conclut son chapitre en parlant de l'activité scientifique comme d'une activité sociale d'élaboration de connaissances.

11. Sur la question de la durée, Berthelot compare plutôt l'approche mertonienne - qui serait davantage diachronique - à celle relativiste - davantage synchronique - et va dans le même sens que nos propos : « [La véritable évaluation scientifique] ne peut-être appréciée, selon Berthelot (2008 : 97), que dans la durée. [...] Autant, ainsi conçu, dans la durée, [le processus d'évaluation] apparait bien régulé par les normes de Merton, autant tout coupe transversale, toute sélection d'un moment, sont susceptibles de voir se multiplier les facteurs adjuvants. »
}

Émulations - Revue des jeunes chercheurs en sciences sociales 


\section{Bibliographie}

Alihan, M. A., [1938] 1964, Social ecology: a critical analysis, New York, Cooper Square Publishers, 267p.

Bergson, H., [1888] 2001, Essai sur les données immédiates de la conscience, Paris, PUF, 180p.

Berthelot, J.-M., 2008, L'emprise du V rai. Connaissance scientifique et modernité, Paris, PUF, 226p.

Boudes, Ph., 2007, «La sociologie de l'environnement, une nouvelle morphologie sociale ? L'apport de Halbwachs à l'étude des relations entre les sociétés et leur milieu naturel », Communication au colloque Autour de Maurice Halbwachs, Université de Reims, octobre 2007.

Boudes, Ph., 2008, L'Environnement, domaine sociologique. La sociologie française au risque de l'environnement, Thèse de doctorat sous la direction de C.-H. Cuin, LAPSAC, Université Bordeaux II, 535p.

Bourg, D., 1993, Présentation de l'ouvrage, in D. Bourg (dir.), La Nature en politique on l'enjeu philosophique de l'écologie, Paris, coédition L'Harmattan/Association Descartes, pp. 6-8

Broswimmer, F. J., [2002] 2003, Ecocide: une brève bistoire de l'extinction en masse des espèces, Paris, Parangon, 260p.

Buttel, F. H., 1978, Environmental sociology: a new paradigm?, The American Sociologist, 13 : 252-256

Buttel, F. H., 1986, Sociologie et environnement : la lente maturation de l'écologie humaine, Revue Internationale des sciences sociales, 109: 359-377

Carson, R., [1962] 1963, Printemps silencieux, Paris, Plon, 287p.

Catton, W. R. \& Dunlap, R.E., 1978, Environmental sociology: a new paradigm, The American Sociologist, 13: 41-49.

Catton, W. R., 2002, Has the Durkheim legacy misled sociology?, in R. E. Dunlap, F. H. Buttel, P. Dickens \& A. Gijswijt (eds), Sociological theory and the environment: classical foundations, contemporary insights, Boulder (Colorado) and New York, Rowman \& Littlefield, pp. 90-118

CMED (Commission Mondiale pour l'Environnement et le Développement), [1987] 1989, Notre avenir à tous, Montréal, éd. Du Fleuve, 434p.

Cuche, D., 2000, Le développement de la la socio-anthropologie des migrations aux Etats-Unis, en ligne sur http://www.reynier.com/Anthro/Interethnique/PDF/Chicago.PDF (consulté en juin 2006), 11p.

Duclos, D., 2006, Introduction : Le mouvement de la question écologique entre 1993 et 2006, in D. Duclos (dir.), Pourquoi tardons-nous temps à devenir écologistes ?, Paris, L'Harmattan, pp. 7-14

Durkheim, E., 1897, La prohibition de l'inceste, L'Année Sociologique, tome 1, 1 ère partie : mémoires originaux

Durkheim, E., 1900, La sociologie en France au XIXe siècle, Revue bleue, 4e série, t XIII, n²0, pp. 609613 et $\mathrm{n}^{\circ} 21$, pp. 647-652, Classiques UQAC [http://classiques.uqac.ca/]

Foster, J. B., 1999, Marx's theory of metabolic rift : classical fundations for environmental sociology, American Journal of Sociology, 105(2): 366-405

Girard, A., 1962, Présentation, in M. Halbwachs, Morphologie Sociale, Paris, Alcan

Grafmeyer, Y. \& Joseph, I., 1979, L'école de Chicago. Naissance de l'écologie urbaine, Paris, éd. du Champ Urbain, 334p.

Émulations - Revue des jeunes chercheurs en sciences sociales 
Grasland, C. 1999, "Problématique du projet 'Morphologie spatiale et morphologie sociale' », proposition de recherche soumise au CNRS et acceptée en septembre 1999.

Gross, M., 2000, Classical Sociology and the Restoration of Nature: The Relevance of Émile Durkheim and Georg Simmel, Organization \& Environment 13 (3):277-291

Gross, M., 2001, Unexpected interactions: Georg Simmel and the observation of nature, Journal of Classical Sociology, 1 (3): 395-414

Halbwachs, M., [1932] 1979, Chicago, expérience ethnique, in Y. Grafmeyer \& I. Joseph, L’École de Chicago, Paris, éd. du Champ urbain

Halbwachs, M., [1938] 1946, Morphologie Sociale, Paris, Alcan, 2ème éd.

Hannigan, J. A., 2006, Environmental sociology, London, Routledge, 194p. + xiv

Hawley, A. H., 1950, Human ecology, New York, Prentice-Hall Inc.

Iijima, N., 1970, Industrial pollution and the community residents' movement: the case of the Minamata disease, Shakaigaku byōron [Japanese Sociological Review], 21 (1): 25-45

Järvikoski, T., 1996, The relation of nature and society in Marx and Durkheim, Acta Sociologica, 39(1): 72-86.

Juan, S., dir., 2007, Actions et enjeux spatiaux en matière d'environnement : de la contestation écologiste aux mesures de protection, Paris, L'Harmattan, coll. Sociologie et environnement, 288p.

Kalaora, B. \& Savoye, A., 1996, Présentation, in F. Le Play, Des Forêts, considérés dans leurs rapports avec la constitution physique du globe et l'économie des sociétés, Fontenay St-Cloud, E.N.S édition

Léger, F., 1989, La Pensée de Georg Simmel : Contribution à l'bistoire des idées en Allemagne au début du XXe siècle, Paris, Kime, 374p. + vi

Luhmann, N., 1993, La société face à l'environnement: une intégration possible?, in D. Bourg (dir.), La Nature en politique on l'enjen philosophique de l'écologie, Paris, coédition L'Harmattan/Association Descartes, pp. 73-85

Meadows, D. H., Meadows, D. L., Randers, J. \& Behrens, W. W., 1972, Halte à la croissance, Paris, Fayard, 315p.

Mitchell, R. E., 2001, Thorstein Veblen, pioneer in environmental sociology, Organization \& Environment, 14 (4): 389-408

Moscovici, S., 2002 De la nature : pour penser l'écologie, éd. Métaillé, 274p. + iii

Park, R. E. \& Burgess E., 1921, Introduction to the Science of Sociology, Chicago, University of Chicago Press

Park, R. E., [1926] 1979, La communauté urbaine : un modèle spatial et un ordre moral, in Y. Grafmeyer \& I. Joseph, L'École de Chicago, Paris, éd. du Champ urbain

Park, R. E., 1950, An autobiographical note, in R. E. Park, Race and culture, Chicago, Free Press, pp. i-ix

Rosa, E. A. \& Richter, L., 2008, Durkheim on the environment: Ex Libris or Ex Cathedra? Introduction to Inaugural Lecture to a Course in Social Science, 1887-1888, Organization and Environment, 21 (2): 182-187

Schnore, L., 1958, Social Morphology and Human Ecology, American Journal of Sociology, 63: 620-634

Simmel, G., 1894, Le problème de la sociologie, Revue de métaphysique et de morale, t. II, pp. 497-504, en ligne sur http://www.ac-nancy-

Émulations - Revue des jeunes chercheurs en sciences sociales 
metz.fr/enseign/philo/textesph/Simmel_Probleme_de_la_sociologie.pdf (consulté le 15 septembre 2008)

Simmel, G., [1923] 1986, Philosophische Kultur. Gesammelte Essais. Berlin, Wagenbach

Simmel, G., [1900] 2007, Philosophie de l'argent, Paris, PUF Quadrige, 662p.

Simmel, G., 1903, Die Großstädte und das Geistesleben, Jabrbuch der Gehestifung, 9: 187-206 [cf. Simmel, G., 2007, Les grandes villes et la vie de l'esprit, Paris, Herne, 59p.]

Simmel, G., [1908] 1979, Digression sur l'étranger, in Y. Grafmeyer \& I. Joseph, L'École de Chicago, Paris, éd. du Champ urbain, pp.53-77

Simmel, G., [1908] 1999, Sociologie : étude sur les formes de la socialisation, Paris, PUF, 756p. + xii

Simmel, G., 1897, Comment les formes sociales se maintiennent, L'Année Sociologique, tome 1, 1ère partie : mémoires originaux

Simmel, G., 1991, Sociologie et épistémologie, Paris, PUF, 238p.

Topalov, Ch., 2006, Maurice Halbwachs et les sociologues de Chicago, Revue francaise de sociologie, 47 (3) : 561-590

Vandenberghe, F., 2000, La sociologie de Georg Simmel, Paris, La Découverte, 123p.

Voltaire (François-Marie Arouet), 1756, Poème sur le désastre de Lisbonne, en ligne sur http://fr.wikisource.org/wiki/Po\%C3\%A8me_sur_le_d\%C3\%A9sastre_de_Lisbonne (consulté fin décembre 2007)

Watier, P., 2003, Georg Simmel, Sociologue, Paris, Circé, 160p.

West, P. C., 1984, Max Weber's human ecology of historical societies, in V. Murvar (ed.), Theory of Liberty, Legitimacy, and Power: New Directions in the Intellectual and Scientific Legacy of Max Weber, London, Routledge \& Kegan Paul, pp. 216-234

Émulations - Revue des jeunes chercheurs en sciences sociales 